\title{
Dynamic Parameter Identification and Analysis of a PHANToM ${ }^{\mathrm{TM}}$ Haptic Device
}

\author{
Amir M. Tahmasebi ${ }^{1}$, Babak Taati ${ }^{2}$, Farid Mobasser $^{2}$, and Keyvan Hashtrudi-Zaad ${ }^{2}$ \\ 1. School of Computing, Queen's University, Kingston, ON, Canada \\ 2. Department of Electrical and Computer Engineering, Queen's University, Kingston, ON, Canada \\ khz@ece.queensu.ca
}

\begin{abstract}
In this paper, the dynamics of a SensAble Technologies PHANToM Premium 1.5 haptic device is experimentally identified and analyzed. Towards this purpose, the dynamic model derived in [1] is augmented with a friction model and is linearly parameterized. The identified model predicts joint torques with over $95 \%$ accuracy and produces an inertia matrix that is confirmed to be positive-definite within the device workspace. In addition, user hand force estimates with and without including the identified dynamics are compared with the measured values. The experiments are also conducted for other typical installation conditions of the device, such as with force sensor mounted at the end-effector, using gimbal and counter-balance weight, and upside-down installation of the device. The contribution of different dynamic terms such as inertial, Coriolis and centrifugal, gravitational, and Coulomb and viscous friction for different installations are demonstrated and discussed. The identified dynamic model can be used for hand force estimation, accurate gravity counter balancing for different installation conditions, and model-based control systems design for haptic simulation and teleoperation applications.
\end{abstract}

\section{INTRODUCTION}

PHANTOM is a force feedback haptic device which was designed by Massie and Salisbury in 1994 and has been commercialized by SensAble Technologies Inc. The PHANToM Premium 1.5 is a desktop tool that allows for the exploration of application areas requiring force feedback in three degreeof-freedom (3-DOF).

Although PHANToM has been widely used in haptic and telerobotic applications [2-4], its functionality is not satisfactory for some high performance applications, partly because the electrical and software subsystems of the PHANToM haptic interface are unknown. To obtain the full functionality of the robot, an accurate dynamic model is the first demand of researchers [5]. Dynamic model design for simulation, control, and contact force observation purposes requires the identification of the dynamic parameters of the manipulator model. Dynamic parameter identification of robotic manipulators has been investigated by researchers [1, 4, 6-11].

The first parameter identification approach is to dismantle various components of a manipulator and to measure and/or calculate the inertial parameters [6-8]. Using computer Aided Design (CAD) models, inertial parameters of various components (links, transmissions, and actuators) can be calculated based on their geometry and the materials used. This method is not easy to implement and there might be difficulties in measuring some of the dynamic parameters. Cavusoglu et al. [1] have previously calculated dynamic parameters of PHANToM based on several simplifying assumptions. However, this technique introduces some inaccuracies due to geometric simplifications in modelling. Moreover, complex dynamic effects such as joint friction are not considered. Mayeda et al. proposed the Sequential Identification Method (SIM) [6] and conducted studies on dynamic models with no redundant parameters [7]. Nakamuro et al. [8] also derived the dynamic models of closedlink manipulators.

Experimental identification of robot dynamics has been reported for industrial manipulators such as excavators [911]. It has been shown that dynamics of manipulators can be linearly parameterized and identified using Least Squares method (LS) [12]. In this work, the dynamic parameter identification of PHANToM Premium 1.5 is experimentally investigated. Our proposed method uses the dynamic structure derived in [1]. The method avoids some of the assumptions made in [1] (e.g. uniformity of link densities, etc.) and can be applied to some other models of PHANToM series devices such as PHANToM Premium 1.0 and 3.0. The method can also be easily applied every time the dynamics of the device is modified. This happens frequently as researchers often use robots in different configurations (e.g. upside-down) or install sensors and tools which changes mass, inertia, and the lengths of different segments of the robot, see Figure 1. In such cases, calculation-based methods for identification are time consuming and are sometimes inaccurate, particularly if the added tools do not have simple geometric shapes.

The sequence of contents in this paper is as follows: The dynamic equations of PHANToM including friction effect are presented and linearly parameterized in Section II. Dynamic parameters of PHANToM under different installations are experimentally identified and verified in Section III. In addition, the contribution of each dynamic component is experimentally demonstrated and discussed. Section IV uses the identified dynamic model for the accurate estimation of hand forces. Section V draws conclusions and discusses future work.

\section{PHANTOM DYNAMIC MODEL}

PHANTOM Premium 1.5 has three degrees of mobility (3 joints) and provides three translational DOF at its end-point 


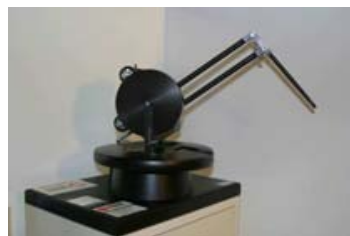

(a)

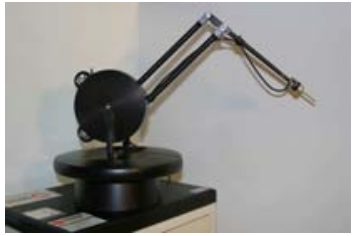

(c)

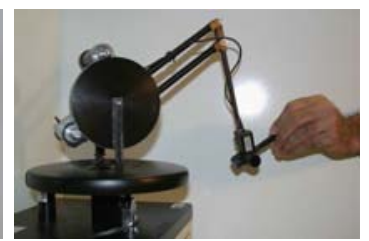

(b)

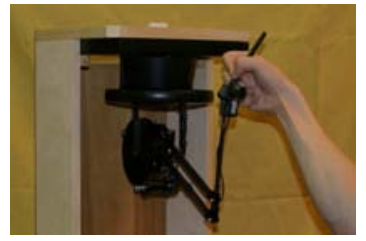

(d)
Fig. 1. PHANToM Premium 1.5 in different typical installation conditions; (a) "Normal", (b) using gimbal and counter-balance weight, (c) with force sensor mounted at the end-effector, and (d) upside-down installation.

as shown in Figure 1(a). Figure 2 shows the schematics of the device with three motors and the corresponding joint angles, $\theta_{1}, \theta_{2}$, and $\theta_{3}$, and a Cartesian frame attached to the end point of the manipulator.
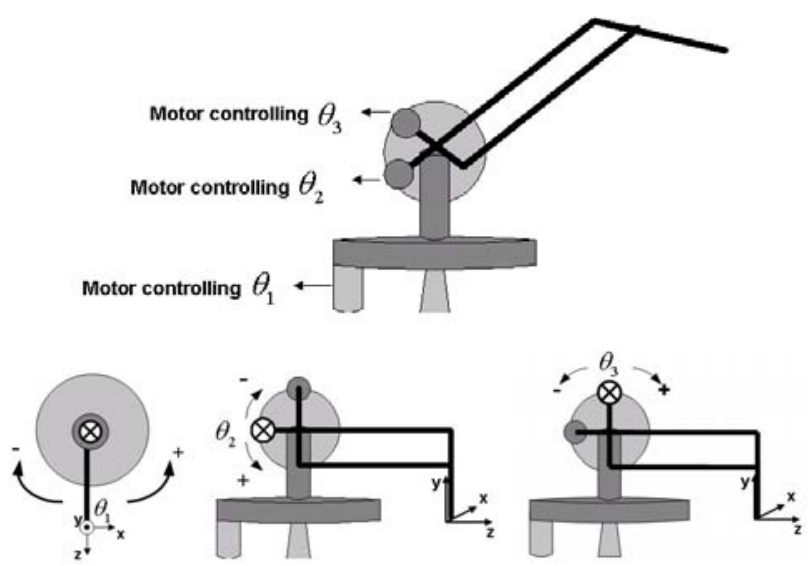

Fig. 2. Schematics of PHANToM with motors, corresponding joint angles and the End-Point (EP) Cartesian frame.

Using the Euler-Lagrange method, Cavusoglu et al. [1] derived the following dynamic structure and equations of motion for PHANToM

$$
\boldsymbol{\tau}=\mathbf{M}(\boldsymbol{\Theta}) \ddot{\Theta}+\mathbf{C}(\boldsymbol{\Theta}, \dot{\Theta}) \dot{\Theta}+\mathbf{N}(\Theta)
$$

or

$$
\begin{gathered}
{\left[\begin{array}{c}
\tau_{1} \\
\tau_{2} \\
\tau_{3}
\end{array}\right]=\left[\begin{array}{ccc}
M_{11} & 0 & 0 \\
0 & M_{22} & M_{23} \\
0 & M_{32} & M_{33}
\end{array}\right]\left[\begin{array}{c}
\ddot{\theta}_{1} \\
\ddot{\theta}_{2} \\
\ddot{\theta}_{3}
\end{array}\right]+} \\
{\left[\begin{array}{ccc}
C_{11} & C_{12} & C_{13} \\
C_{21} & 0 & C_{23} \\
C_{31} & C_{32} & 0
\end{array}\right]\left[\begin{array}{c}
\dot{\theta}_{1} \\
\dot{\theta}_{2} \\
\dot{\theta}_{3}
\end{array}\right]+\left[\begin{array}{c}
0 \\
N_{2} \\
N_{3}
\end{array}\right]}
\end{gathered}
$$

where $\mathbf{M}, \mathbf{C}$, and $\mathbf{N}$ represent the inertial matrix, Coriolis and centrifugal matrix, and gravitational vector, respectively, defined in terms of the inertial and kinematic properties of the device individual segments [1]. Here, $\boldsymbol{\tau}=\left[\begin{array}{lll}\tau_{1} & \tau_{2} & \tau_{3}\end{array}\right]^{T}$ and $\boldsymbol{\Theta}=\left[\begin{array}{lll}\theta_{1} & \theta_{2} & \theta_{3}\end{array}\right]^{T}$ are the torque command voltage vector sent to the amplifier box and the joint angle vector read by the encoders, respectively. The reader should note that in [1], $\tau$ represents the vector of induced motor torques rather than the torque command voltage. As the electric dynamics of the motors and the amplifier box are assumed to be much faster than the mechanical dynamics of the motors and the linkages, the torque command voltages are proportional to the motor induced torques. Experimental results have shown that actual motor torques can be found by scaling down the torque command voltage $\tau$ by a factor of $\alpha=2.73$. In the sequel, "torque" refers to the torque command voltage vector.

To identify the device dynamics, eq. (2) is linearly parameterized as:

$$
\tau=\mathbf{Y}(\Theta, \dot{\Theta}, \ddot{\Theta}) \pi
$$

where $\mathbf{Y}$ is the regressor matrix and $\pi$ is the vector of 8 unknown parameters, defined as:

$$
\boldsymbol{\pi}=\left[\begin{array}{c}
\boldsymbol{\pi}_{d} \\
-- \\
\boldsymbol{\pi}_{g}
\end{array}\right]=\left[\begin{array}{c}
\frac{1}{8}\left(4 I_{a y y}+4 I_{a z z}+8 I_{b a y y}+I_{b e y y}+4 I_{b e z z}+4 I_{c y y}+4 I_{c z z}\right. \\
\left.+4 I_{d f y y}+4 I_{d f z z}+L_{1}^{2} m_{c}+L_{2}^{2} m_{a}+4 L_{3}^{2} m_{c}+4 L_{1}^{2} m_{a}\right) \\
\frac{1}{8}\left(4 I_{b e y y}-4 I_{b e z z}+4 I_{c y y}-4 I_{c z z}+4 L_{1}^{2} m_{a}+L_{1}^{2} m_{c}\right) \\
\frac{1}{8}\left(4 I_{a y y}-4 I_{a z z}+4 I_{d f y y}-4 I_{d f z z}-L_{2}^{2} m_{a}-4 L_{3}^{2} m_{c}\right) \\
L_{1}\left(L_{2} m_{a}+L_{3} m_{c}\right) \\
\frac{1}{4}\left(4 I_{b e x x}+4 I_{c x x}+4 L_{1}^{2} m_{a}+L_{1}^{2} m_{c}\right) \\
\frac{1}{4}\left(4 I_{a x x}+4 I_{d f x x}+L_{2}^{2} m_{a}+4 L_{3}^{2} m_{c}\right) \\
----------------------- \\
\frac{g}{2}\left(2 L_{1} m_{a}+2 L_{5} m_{b e}+L_{1} m_{c}\right) \\
\frac{g}{2}\left(L_{2} m_{a}+2 L_{3} m_{c}-2 L_{6} m_{d f}\right)
\end{array}\right]
$$

where $\boldsymbol{\pi}_{d}$ and $\boldsymbol{\pi}_{g}$ represent dynamic and gravitational parameter vectors, respectively. The inertial and kinematic parameters $L_{1}, L_{2}, L_{3}, L_{5}, L_{6}, I_{a x x}, I_{a y y}, I_{a z z}, I_{c x x}, I_{c y y}$, $I_{c z z}, I_{b a y y}, I_{b e x x}, I_{b e y y}, I_{b e z z}, I_{d f x x}, I_{d f y y}, I_{d f z z}, m_{a}, m_{c}$, $m_{b e}$, and $m_{d f}$ are defined in [1] and $g$ is the acceleration gravity. The $\mathbf{Y}$ matrix is given in (5), where $s_{i}, c_{i}, s_{i j}$, $c_{i j}, s_{2 . i}$, and $c_{2 . i}, i, j=1,2,3$, stand for $\sin \left(\theta_{i}\right), \cos \left(\theta_{i}\right)$, $\sin \left(\theta_{i}-\theta_{j}\right), \cos \left(\theta_{i}-\theta_{j}\right), \sin \left(2 \theta_{i}\right)$, and $\cos \left(2 \theta_{i}\right)$, respectively.

\section{A. Inclusion of Friction Model}

The following classic model is used to include friction effect in the dynamic model of the device as:

$$
\boldsymbol{\tau}_{f}=\boldsymbol{\tau}_{f c} \operatorname{sgn}(\dot{\boldsymbol{\Theta}})+\boldsymbol{\tau}_{f v} \dot{\boldsymbol{\Theta}}
$$

or

$$
\boldsymbol{\tau}_{f}=\left[\begin{array}{c}
\tau_{f_{1}} \\
\tau_{f_{2}} \\
\tau_{f_{3}}
\end{array}\right]=\left[\begin{array}{c}
\pi_{f c_{1}} \operatorname{sgn}\left(\dot{\theta}_{1}\right)+\pi_{f v_{1}} \dot{\theta}_{1} \\
\pi_{f c_{2}} \operatorname{sgn}\left(\dot{\theta}_{2}\right)+\pi_{f v_{2}} \dot{\theta}_{2} \\
\pi_{f c_{3}} \operatorname{sgn}\left(\dot{\theta}_{3}\right)+\pi_{f v_{3}} \dot{\theta}_{3}
\end{array}\right]=\mathbf{Y}_{f} \boldsymbol{\pi}_{f}(7)
$$

where $\pi_{f c_{i}}$ and $\pi_{f v_{i}}$ are the Coulomb and viscous friction coefficients for joint " $i$ ", and $\boldsymbol{\tau}_{f c}=\operatorname{diag}\left(\tau_{f c_{i}}\right), \boldsymbol{\tau}_{f v}=$ $\operatorname{diag}\left(\tau_{f v_{i}}\right), i=1,2,3$. $\mathbf{Y}_{f}$ and $\boldsymbol{\pi}_{f}$ are defined as: 


$$
\mathbf{Y}^{T}=\left[\begin{array}{c}
\mathbf{Y}_{d}^{T} \\
-- \\
\mathbf{Y}_{g}^{T}
\end{array}\right]=\left[\begin{array}{ccc}
\ddot{\theta}_{1} & 0 & 0 \\
\ddot{\theta}_{1} c_{2.2}-2 \dot{\theta}_{1} \dot{\theta}_{2} s_{2} c_{2}-\dot{\theta}_{1} \dot{\theta}_{2} s_{2.2} & \dot{\theta}_{1}^{2} s_{2.2} & 0 \\
\ddot{\theta}_{1} c_{2.3}-2 \dot{\theta}_{1} \dot{\theta}_{3} s_{3} c_{3}-\dot{\theta}_{1} \dot{\theta}_{3} s_{2.3} & 0 & \dot{\theta}_{1}^{2} s_{2.3} \\
\ddot{\theta}_{1} c_{2} s_{3}-\dot{\theta}_{1} \dot{\theta}_{2} s_{2} s_{3}+\dot{\theta}_{1} \dot{\theta}_{3} c_{2} c_{3} & -\frac{1}{2} \ddot{\theta}_{3} s_{23}+\frac{1}{2} \dot{\theta}_{1}^{2} s_{2} s_{3}+\frac{1}{2} \dot{\theta}_{3}^{2} c_{23} & -\frac{1}{2} \ddot{\theta}_{2} s_{23}-\frac{1}{2} \dot{\theta}_{1}^{2} c_{2} c_{3}+\frac{1}{2} \dot{\theta}_{2}^{2} c_{23} \\
0 & \ddot{\theta}_{2} & 0 \\
0 & 0 & \ddot{\theta}_{3} \\
-------------- & 0 \\
0 & ---c---1 & s_{3}
\end{array}\right]
$$

$$
\begin{gathered}
\mathbf{Y}_{f}=\left[\begin{array}{cccccc}
\dot{\theta}_{1} & 0 & 0 & \operatorname{sgn}\left(\dot{\theta}_{1}\right) & 0 & 0 \\
0 & \dot{\theta}_{2} & 0 & 0 & \operatorname{sgn}\left(\dot{\theta}_{2}\right) & 0 \\
0 & 0 & \dot{\theta}_{3} & 0 & 0 & \operatorname{sgn}\left(\dot{\theta}_{3}\right)
\end{array}\right] \\
\boldsymbol{\pi}_{f}=\left[\begin{array}{llllll}
\pi_{f v_{1}} & \pi_{f v_{2}} & \pi_{f v_{3}} & \pi_{f c_{1}} & \pi_{f c_{2}} & \pi_{f c_{3}}
\end{array}\right]^{T}
\end{gathered}
$$

where $\operatorname{sgn}$ denotes the signum function. Considering friction effects in the dynamic model, the total $\mathbf{Y}$ and $\boldsymbol{\pi}$ expand to:

$$
\boldsymbol{\pi}=\left[\begin{array}{c}
\boldsymbol{\pi}_{d} \\
-- \\
\boldsymbol{\pi}_{g} \\
-- \\
\boldsymbol{\pi}_{f}
\end{array}\right]
$$

and

$$
\mathbf{Y}_{[3 \times 14]}=\left[\begin{array}{lll}
\mathbf{Y}_{d_{[3 \times 6]}} & \mathbf{Y}_{g_{[3 \times 2]}} & \mathbf{Y}_{f_{[3 \times 6]}}
\end{array}\right]
$$

\section{EXPERIMENTAL RESULTS}

In this section, the results of experimental parameter identification are discussed and PHANToM dynamics are analyzed. The experimental setup consists of a PHANToM Premium 1.5, an ATI Nano- $17^{\mathrm{TM}}$ force/torque sensor and a real-time open-architecture control system developed inhouse for full functionality of the robot. The developed open-architecture platform ${ }^{1}$ that utilizes PHANToM amplifier box uses a Quanser Q8 ${ }^{\mathrm{TM}}$ data acquisition board and a WinCon $^{\mathrm{TM}} / \mathrm{RTX}^{\mathrm{TM}}$ real-time system, which links well with MATLAB Real-Time Workshop ${ }^{\mathrm{TM}}$. The sensory information provided by the setup are the three motor angles read by encoders, and six end-point generalized forces provided by the force sensor in three Cartesian directions. Data collection and control processes run at the rate of $1 \mathrm{kHz}$. Joint velocities are estimated from joint angles by using a highpass filter numerical differentiator. Numerical calculation of joint accelerations significantly amplifies the noise and is not recommended. Instead, a filtering technique proposed in [13] is used to eliminate the need for acceleration measurement. To this end, the system total dynamic model (3), (10), and (11) is passed through a strictly stable low-pass filter with transfer function such as $L(s)=\frac{\omega}{\omega+s}, \omega>0$ to obtain

$$
\boldsymbol{\tau}_{L}=\mathbf{Y}_{L}(\boldsymbol{\Theta}, \dot{\Theta}) \boldsymbol{\pi}
$$

where $\mathbf{Y}_{L}(\boldsymbol{\Theta}, \dot{\boldsymbol{\Theta}})$ and $\tau_{L}$ are filtered $\mathbf{Y}$ and $\boldsymbol{\tau}$. The regressor matrix $\mathbf{Y}_{L}$ is a function of only joint angle $\Theta$ and joint

\footnotetext{
${ }^{1}$ For more information, refer to http://www.quanser.com
}

velocity $\dot{\Theta}$, since the filtered acceleration can be written as a function of joint velocity and filtered velocity; that is $\ddot{\theta}_{i L}=\omega\left(\dot{\theta}_{i}-\dot{\theta}_{i L}\right), i=1,2,3$. The elements of $\mathbf{Y}_{L}$ are given in (13) where the subscript " $L$ " means that the argument has passed through filter $L(s)$. By designing the filter such that its cut-off frequency lies between the system bandwidth and the noise frequency, it is possible to attenuate the degrading effects of the input and measurement noise on the identification performance.

For identification experiments, a simple PD controller for angular position tracking was designed using Simulink ${ }^{\mathrm{TM}}$. The desired joint trajectory inputs were chosen for higher level of system excitation [14] needed for better convergence of the identified parameters to their true values [12]. Towards this end, joint trajectories were constructed from several sinusoids with various frequencies and amplitudes for each joint such that the robot workspace was not violated. As the sampling frequency was $1 \mathrm{kHz}$, the chosen sine frequencies were well below the Nyquist rate $500 \mathrm{~Hz}$.

The experiments were all conducted for a time duration of 40 seconds. Since the low pass filter was implemented using a memory unit in the discrete time domain, the first few seconds of the filtered data were discarded to allow for the output of the filter to converge. The first half of the collected data were utilized to identify the dynamic parameters. For cross validation, the estimated parameters were then employed to predict joint torques with the second half of the data. Since filtered values of torque and joint velocities were used in the estimation process, the filtered torques were compared with their predicted values.

\section{A. Effect of Friction}

In the first experiment, friction parameters were not included and only 8 dynamic parameters, $\boldsymbol{\pi}_{d}$ and $\boldsymbol{\pi}_{g}$, were identified. Figure 3 compares the actual filtered torque $\tau_{L}$ with two estimated filtered torques $\hat{\tau}_{L}=\mathbf{Y}_{L} \hat{\boldsymbol{\pi}}$ using the identified parameters and the measured parameters provided in [1]. The large errors between the actual and the estimated filtered torques for joints 1 and 2 were expected to represent the unaccounted friction in joints and the power transmission system. For clarification, the filtered torque prediction errors, $\tau_{L}-\hat{\tau}_{L}$, are plotted versus joint velocities in Figure 4. As it can be observed, substantial friction exists in all joints, especially in joint 1 .

Therefore, six additional friction parameters were included in the dynamic model as mentioned in Section II-A and 14 $\pi$ parameters were identified in total. The 14 identified parameters and the 8 parameters calculated from the measured values in [1] are shown in the second and the third columns 


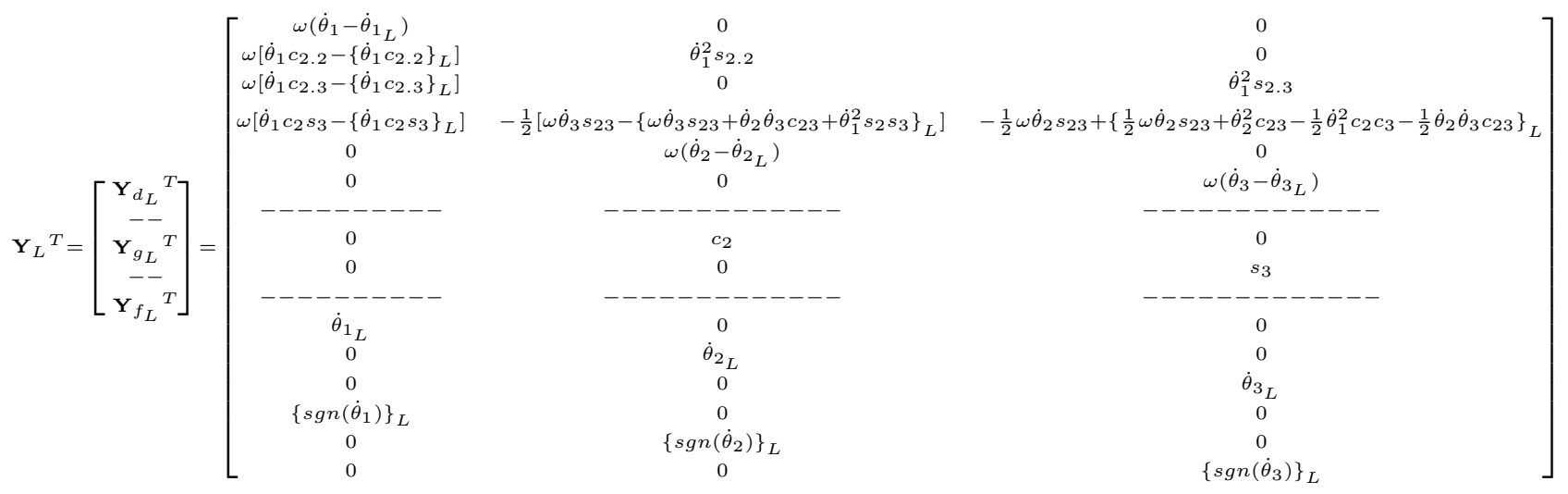
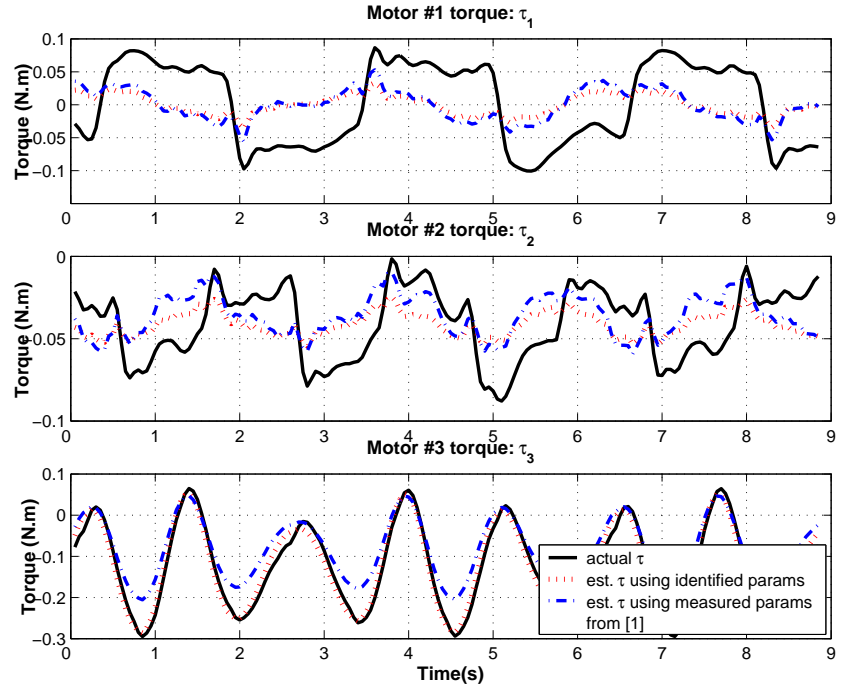

Fig. 3. Actual and estimated $\tau$; the solid black line shows the real filtered torque, the dotted red line shows the predicted filtered torque using estimated $\pi$ parameters (without friction in the dynamic model), and the dash-dot blue line shows the predicted filtered torque using values given in [1].
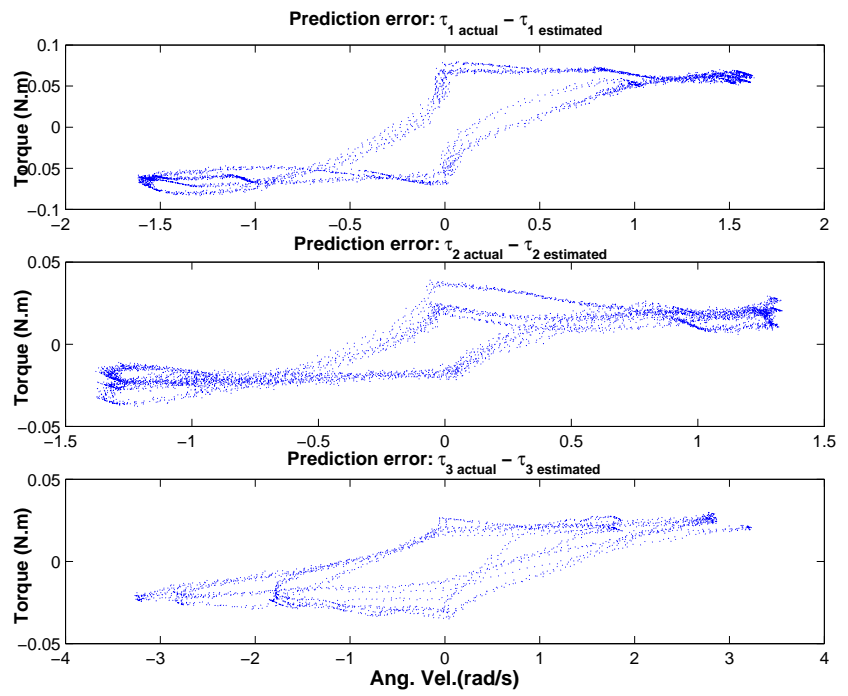

Fig. 4. Torque estimation error versus joint angular velocity (without friction in the dynamic model). of Table I. The two parameter sets have the same signs and are relatively close, which to some extent validates the assumptions made in [1]. Figure 5 illustrates the estimated torques with identified parameters after including friction in the model. It can be observed that the estimated torque follows the actual value closely.
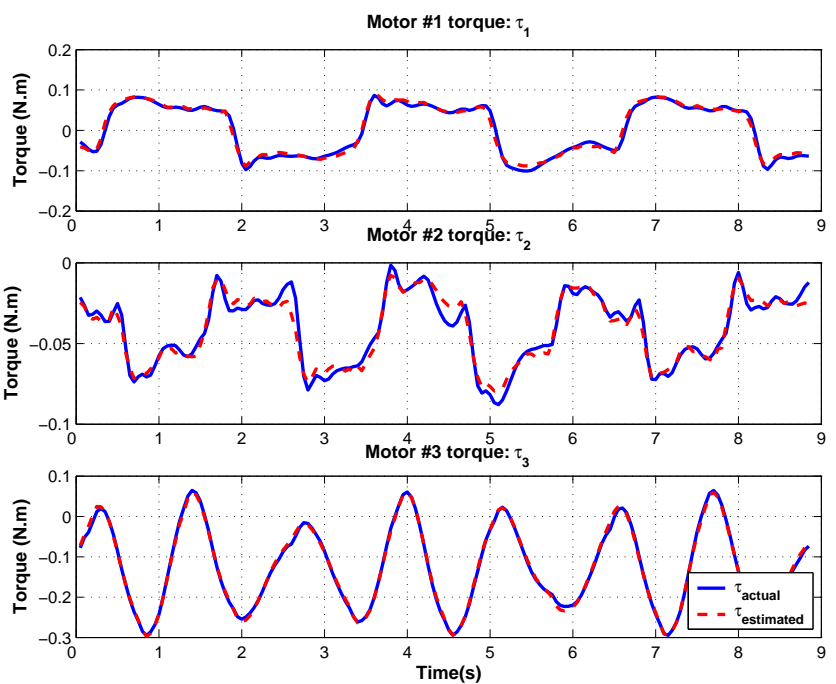

Fig. 5. Actual and estimated $\tau$ using identified $\pi$ parameters (with friction in the dynamic model).

In order to grasp a better view on the effect of each friction term, Coulomb and viscous terms are illustrated separately in Figure 6. The first joint has the most significant friction among all three. The effect of Coulomb friction is unanimously higher than that of the viscous friction for such speed of motion.

\section{B. Various Configurations}

Four additional installation configurations were considered for the PHANToM as described below:

1) Gimbal and counter balance weight mounted (GC): The gimbal and the counter balance weight supplied by the manufacturer are mounted on the PHANToM for additional passive DOF as shown in Figure 1(b).

2) Force sensor mounted (FS): For Haptic applications, measured hand force can be used in the design of transparent controller (Figure 1(c)). 

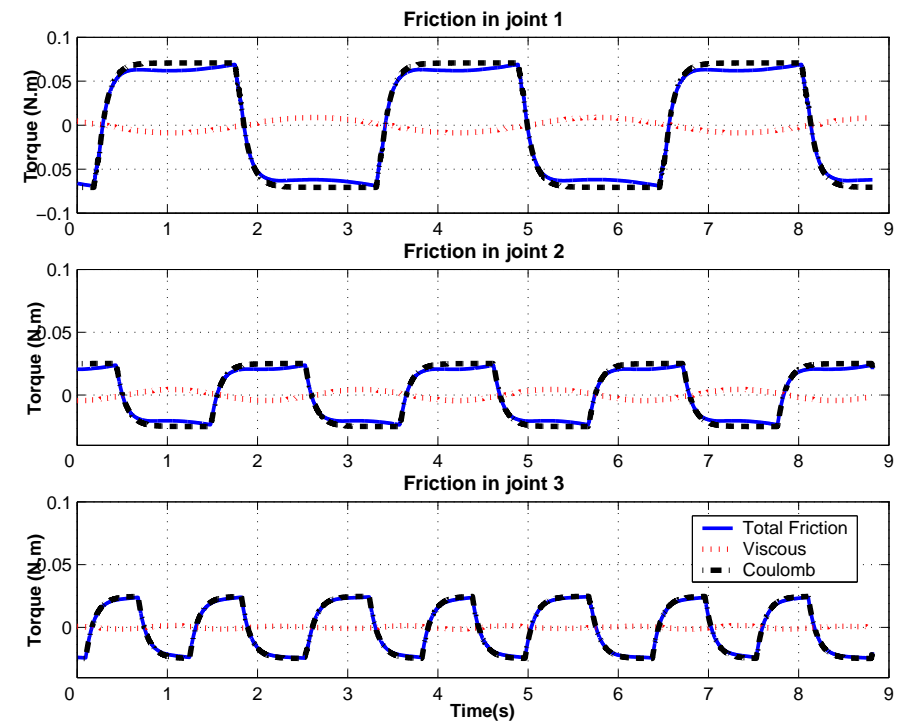

Fig. 6. Contribution of viscous and Coulomb friction terms in overall friction, in all three joints.

\begin{tabular}{|c||c|c|c|c|c|c|}
\hline$\hat{\pi}$ & {$[1]$} & Normal & GC & FS & USD & USD+GC \\
\hline$\hat{\pi}_{1}$ & 0.0076 & 0.0039 & 0.0093 & 0.0028 & 0.0033 & 0.0083 \\
\hline$\hat{\pi}_{2}$ & 0.0030 & 0.0037 & 0.0121 & 0.0059 & 0.0044 & 0.0090 \\
\hline$\hat{\pi}_{3}$ & -0.0011 & -0.0011 & -0.0032 & -0.0021 & -0.0014 & -0.0020 \\
\hline$\hat{\pi}_{4}$ & 0.0025 & 0.0019 & 0.0252 & 0.0082 & 0.0018 & 0.0251 \\
\hline$\hat{\pi}_{5}$ & 0.0066 & 0.0057 & 0.0206 & 0.0095 & 0.0078 & 0.0174 \\
\hline$\hat{\pi}_{6}$ & 0.0025 & 0.0026 & 0.0097 & 0.0031 & 0.0035 & 0.0086 \\
\hline$\hat{\pi}_{7}$ & -0.0445 & -0.0525 & 0.0735 & 0.1279 & 0.0580 & -0.0453 \\
\hline$\hat{\pi}_{8}$ & -0.2015 & -0.3002 & 0.1270 & -0.1886 & 0.2969 & -0.3070 \\
\hline$\hat{\pi}_{9}$ & - & -0.0057 & -0.0074 & -0.0025 & -0.0037 & -0.0003 \\
\hline$\hat{\pi}_{10}$ & - & -0.0035 & 0.0003 & -0.0009 & -0.0004 & 0.0015 \\
\hline$\hat{\pi}_{11}$ & - & -0.0005 & 0.0031 & 0.0012 & 0.0025 & 0.0020 \\
\hline$\hat{\pi}_{12}$ & - & 0.0707 & 0.0716 & 0.0739 & 0.0712 & 0.0665 \\
\hline$\hat{\pi}_{13}$ & - & 0.0251 & 0.0228 & 0.0255 & 0.0225 & 0.0199 \\
\hline$\hat{\pi}_{14}$ & - & 0.0248 & 0.0247 & 0.0273 & 0.0221 & 0.0238 \\
\hline
\end{tabular}

TABLE I

ESTIMATED $\pi$ PARAMETERS OF DIFFERENT CONFIGURATIONS; GC: GiMBAL + COUNTER BALANCE, FS: ForCE SENSOR, USD:

UPSIDE-DOWN, USD + GC: UPSIDE-DOWN + GIMBAL + COUNTER BALANCE.

3) Upside-down (USD): One of the most common configurations of PHANToM, especially for medical simulators, is to install PHANToM upside-down for better ergonomics.

4) Upside-down plus gimbal and counter balance weight $(U S D+G C)$ : Figure 1(d), shows a PHANToM 1.5 used in an ultrasound training simulator developed by the authors [15]. For this particular application, the gimbal and the counter balance weight supplied by the manufacturer are mounted on the device. Therefore, the dynamic parameters for this configuration has also been identified.

\section{Observation}

The $\pi$ parameters estimated for each configuration are listed in Table I. Note that for all configurations, friction was included in the dynamic model. The variance of the estimates were in the range of $10^{-6}$ and $10^{-9}$. Note that the dynamic parameters signifying the dynamics from motor torques to joint angles can be found by dividing the $\pi$ parameters in Table I by $\alpha=2.73$. The followings can be observed from Table I:

(1) $\pi_{7}$ and $\pi_{8}$ correspond to the gravitational effect. By adding the gimbal and counter balance weight, these parameters become very small. This verifies that the counter balance compensates well for the gravitational effect.

(2) Comparing $\pi_{1}$ to $\pi_{6}$ in "Normal" and GC columns shows that adding gimbal and counter balance weight adds substantially to the device inertia.

(3) Comparing $\pi_{1}$ to $\pi_{8}$ parameters for Normal and USD configurations shows that changing the PHANToM installation to upside-down does not change the dynamic parameters except for the sign of the gravitational parameters $\pi_{7}$ and $\pi_{8}$. This confirms the fact that in USD configuration, the arm moves downward when it is not activated. In Normal installation, the arm moves in opposite direction.

(4) By adding the gimbal and counter balance weight to the upside-down configuration, $\pi_{7}$ and $\pi_{8}$ change their signs. But the high absolute values imply that the counter balance weight is not compensating satisfactorily for the gravitational terms. Therefore, either a new counter balance weight needs to be designed or active control should compensate for gravity in USD configuration.

(5) The gravitational parameters $\pi_{7}$ and $\pi_{8}$, change from -0.525 , and -0.3002 to 0.1279 and -0.1886 for force sensor mounted configuration. Reduction in absolute values implies that force sensor acts as a counter balance weight.

(6) The viscous parameters $\pi_{9}-\pi_{11}$, are small confirming that there is no significant viscous friction in PHANToM joints.

\section{Validation}

\begin{tabular}{|c||c||c||c||c||c|}
\hline RMS & Normal & GC & FS & USD & USD + GC \\
\hline$\tau_{1}$ & 1.53 & 2.42 & 2.91 & 1.89 & 3.70 \\
\hline$\tau_{2}$ & 1.15 & 0.84 & 1.09 & 0.81 & 1.29 \\
\hline$\tau_{3}$ & 0.10 & 5.02 & 1.70 & 0.21 & 2.88 \\
\hline
\end{tabular}

TABLE II

RMS VALUES OF THE ACTUAL AND ESTIMATED TORQUE ERRORS IN PERCENT FOR DIFFERENT CONFIGURATIONS; GC: GIMBAL + COUNTER BALANCE, FS: ForCE SENSOR, USD: Up-SidE-DOWN, USD + GC:

UPSIDE-DOWN + GIMBAL + COUNTER BALANCE.

For the entire workspace of the robot and for all installations, the estimate of inertia matrix were confirmed to be positive definite with a minimum eigenvalue of $1.8 \times 10^{-3}$.

The RMS values [11] of the filtered torque estimation errors for all configurations are listed in Table II. The GC and USD + GC configurations have the most significant errors among all others. This can be due to the movement of the gimbal since it is not possible to rigidly fix the gimbal to the 
robot arm while the arm is moving. Any small movement of the gimbal can noticeably change the dynamics of the robot.

\section{E. Dynamic Dissection}

In order to further analyze system dynamics, the estimated $\pi$ parameters are used to calculate joints torques and the corresponding contributions of inertia, Coriolis and centrifugal, gravity and friction terms for each configuration. From Figures 7-11 that illustrate the contribution of these terms for each configuration, the followings can be observed and concluded:
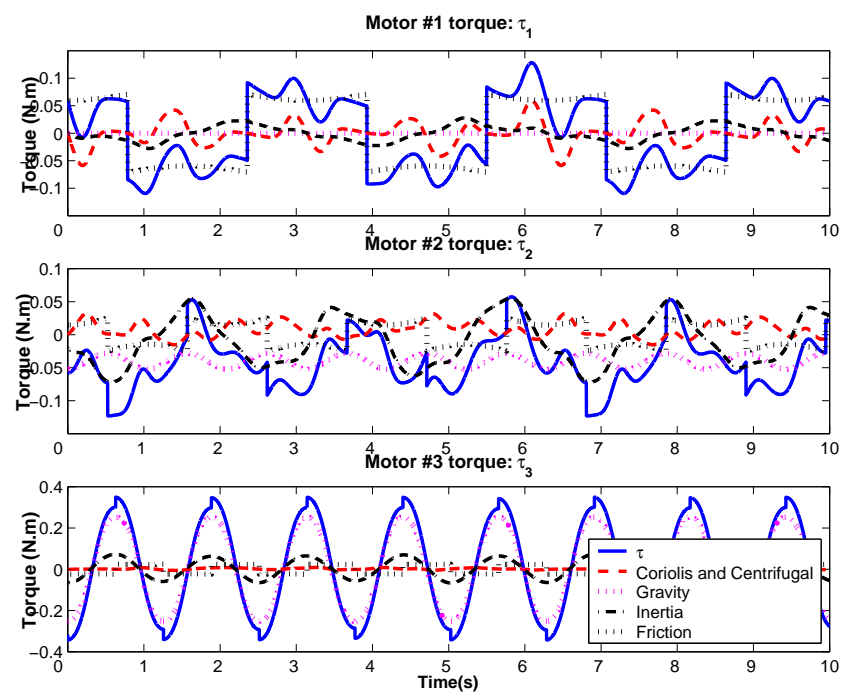

Fig. 7. Contribution of inertia, Coriolis and centrifugal, gravity, and friction in joint torque $\tau$ (Normal configuration).
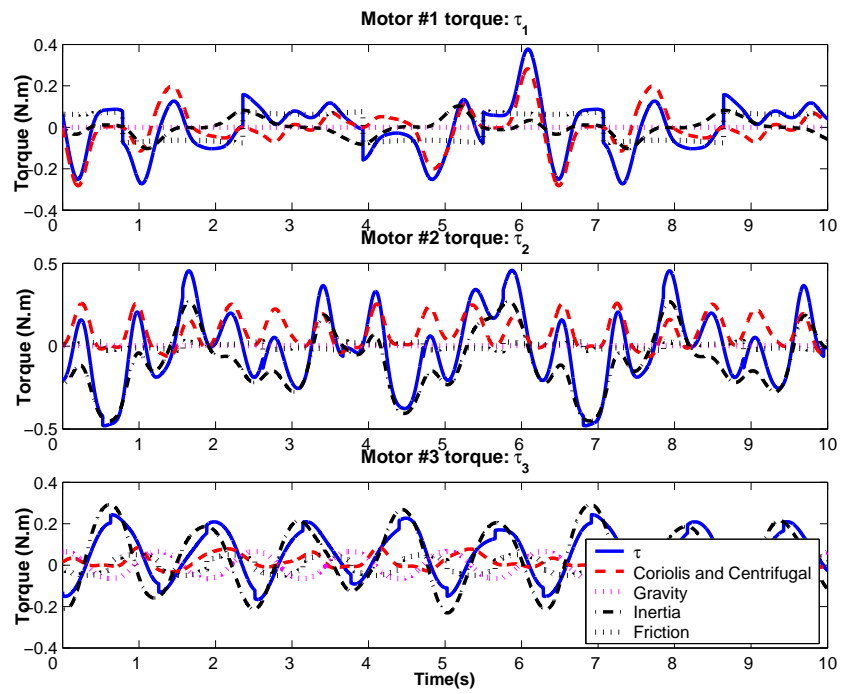

Fig. 8. Contribution of inertia, Coriolis and centrifugal, gravity, and friction in joint torque $\tau$ (gimbal and counter balance mounted).

(1) Joint 1: For all installations, the most significant contribution comes from friction, and Coriolis and
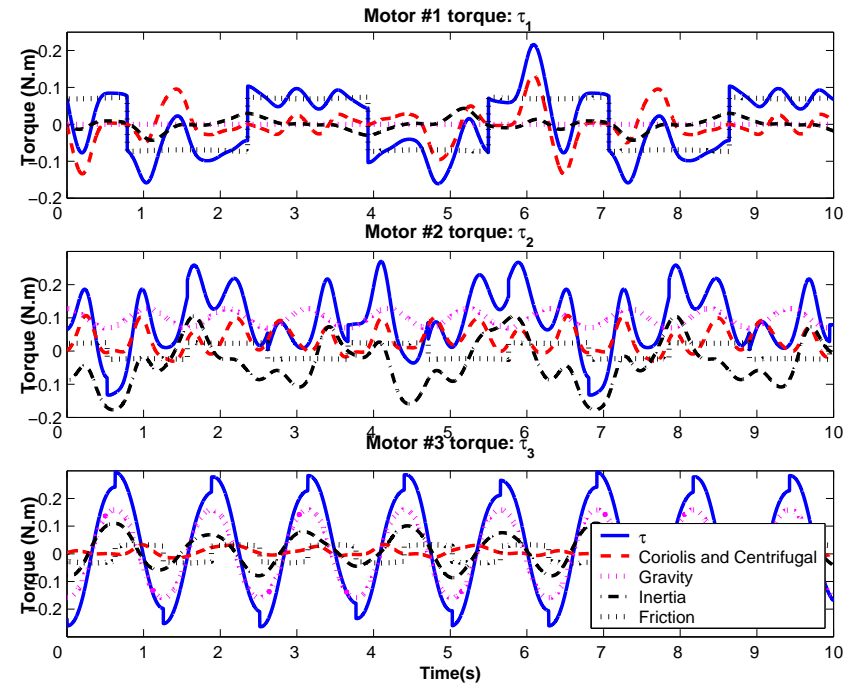

Fig. 9. Contribution of inertia, Coriolis and centrifugal, gravity, and friction in ioint toraue $\tau$ (Force sensor mounted).
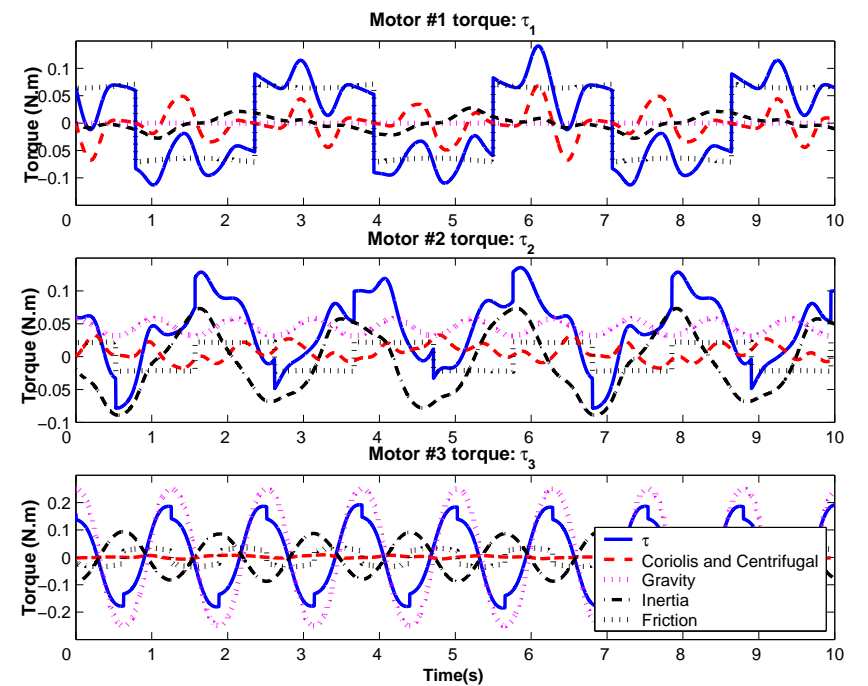

Fig. 10. Contribution of inertia, Coriolis and centrifugal, gravity, and friction in joint torque $\tau$ (Upside-down configuration).

centrifugal terms.

(2) Joint 2: The main contribution comes from inertial term and then Coriolis and centrifugal terms.

(3) Joint 3: In general, the contribution of gravity in torque is the most significant in joint 3 compared to other two. For Normal installation, adding extra mass such as force sensor cuts down the effect of gravity by half and adding gimbal and counter balance weight almost practically nulls the effect of gravity.

\section{FORCE ESTIMATION}

Haptic interfaces produce sense of contact between human body and virtual objects by displaying a bi-directional force channel. To design transparent controllers high bandwidth contact forces need to be measured or estimated. Using an 

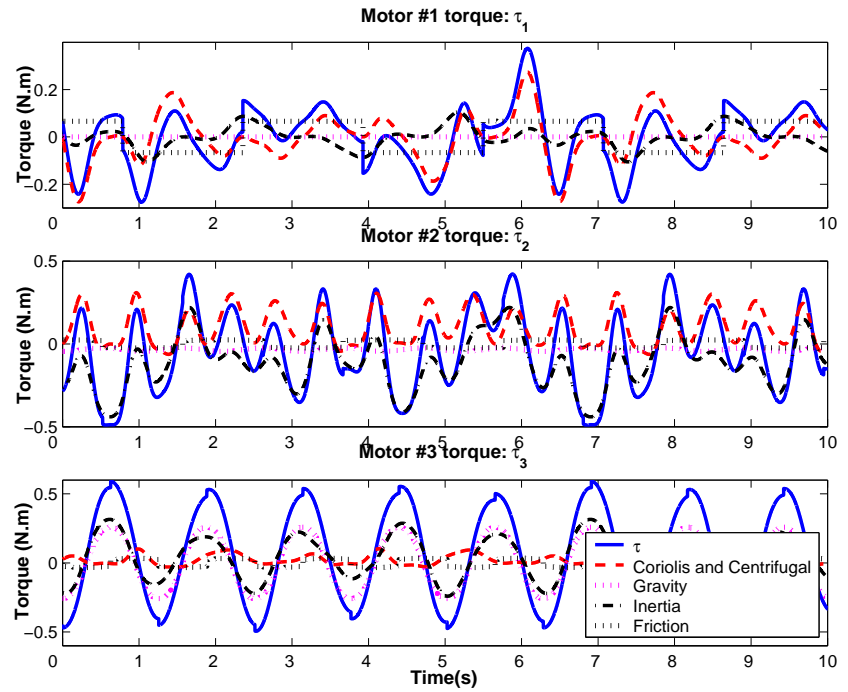

Fig. 11. Contribution of inertia, Coriolis and centrifugal, and gravity in total amount of $\tau$ (Upside-down configuration plus gimbal and counter balance mounted).

accurate estimate of PHANToM, hand forces are estimated and compared to the output of an ATI Nano- $17^{\mathrm{TM}}$ force sensor, mounted at the End Point (EP) of the device as shown in Figure 1(c).

Using the device Jacobian provided in [1], the hand force can be estimated from system dynamics as:

$$
\hat{\mathbf{F}}_{e x t}=\frac{1}{\alpha}\left(\mathbf{J}_{p}\right)^{-T}\left(\boldsymbol{\tau}-\mathbf{Y} \hat{\boldsymbol{\pi}}-\mathbf{J}_{o} \boldsymbol{\tau}_{e x t}\right)
$$

where

$$
\mathbf{J}=\left[\begin{array}{c}
\mathbf{J}_{p} \\
-- \\
\mathbf{J}_{o}
\end{array}\right]=\left[\begin{array}{ccc}
l_{1} c_{2}+l_{2} s_{3} & 0 & 0 \\
0 & l_{1} c_{23} & 0 \\
0 & -l_{1} s_{23} & l_{2} \\
--- & --- & --- \\
0 & 0 & -1 \\
c_{3} & 0 & 0 \\
s_{3} & 0 & 0
\end{array}\right]
$$

is the Jacobian in the EP frame (see Figure 2) and $\tau_{\text {ext }}$ is the external torque measured by the Nano-17 sensor. Note that i) forces are scaled by $\alpha$ in order to accommodate the gain of electronics, and ii) $\hat{\mathbf{F}}_{\text {ext }}$ can be found without the need for $\tau_{\text {ext }}$ by using pseudo inverse of $\mathbf{J}$. The measured forces provided by the force sensor are also transformed into the EP frame as well.

Figure 12 compares the actual force in $\mathrm{Y}$ direction to the estimated forces obtained with (eq. (14)) and without using the dynamic model (i.e. $\hat{\mathbf{F}}_{\text {ext }}=\frac{1}{\alpha}\left(\mathbf{J}_{p}\right)^{-T}\left(\boldsymbol{\tau}-\mathbf{J}_{o} \boldsymbol{\tau}_{\text {ext }}\right)$ ). As it can be seen, under dynamic condition especially when changing the direction of motion, force estimation with dynamic model produces slightly better results.

\section{CONClusion And Future Work}

We have successfully identified the dynamics of a SensAble Technologies PHANToM Premium 1.5 using an experimental method. The advantages of our method over the
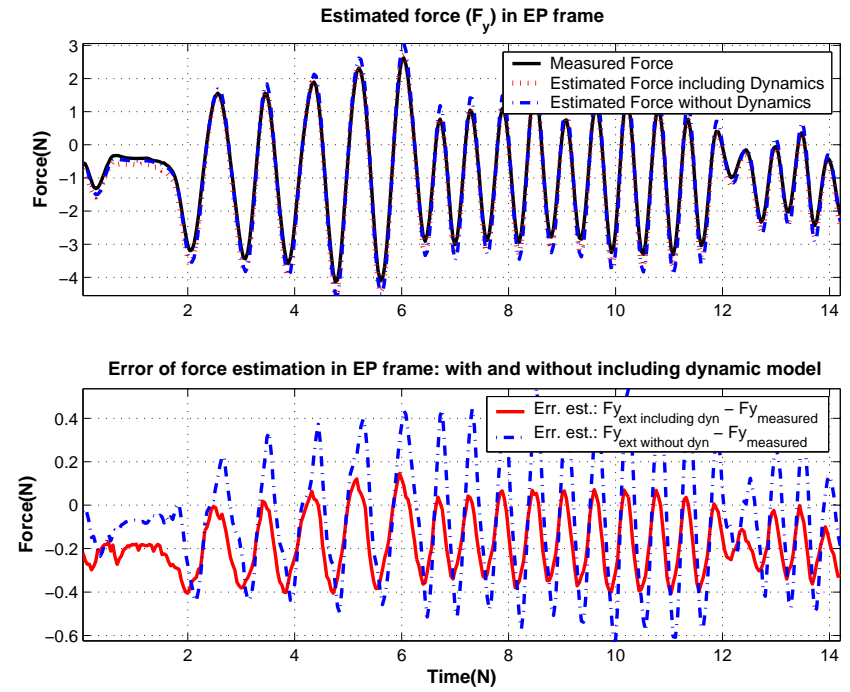

Fig. 12. (a) Measured and estimated forces expressed in EP frame; $F_{y}$ in ydirection of EP frame, and the corresponding error (b) including dynamics, and (c) without including dynamic model.

previously developed CAD-based method are the inclusion of an efficient yet simple friction model, the ease of use for handling alterations to the device dynamics, and applicability to other models of Phantom Premium devices. Free motion experimental results demonstrated noticable Coulomb friction in all three joints, especially in joint 1 . The haptic device dynamics for different installation configurations, such as with force/torque sensor, with gimbal and counter balance weight, and upside-down, were also identified, validated and analyzed. Estimated dynamic parameters were able to predict joint torques and human hand contact forces with over $95 \%$ accuracy. The identified dynamic model will be utilized in inverse dynamics based controllers for haptic and teleoperation applications.

\section{REFERENCES}

[1] M. C. Cavusoglu and D. Feygin, "Kinematics and dynamics of phantom(tm) model 1.5 haptic interface," UC Berkeley ERL Memo M01/15, 2001. [Online]. Available: http://vorlon.cwru.edu/

[2] M. C. Cavusoglu, A. Sherman, and F. Tendick, "Design of bilateral teleoperation controllers for haptic exploration and telemanipulation of soft environments," IEEE Trans. Rob. \& Auto., vol. 18, pp. 641-647, 2002.

[3] F. Mobasser and K. Hashtrudi-Zaad, "Implementation of a rate mode impedance reflecting teleoperation controller on a haptic simulation system," Proc. IEEE Intl. Conf. Rob. \& Auto., pp. 1974-1979, 2004.

[4] M. Tavakoli, R. V. Patel, and M. Moallem, "Design issues in a haptics-based master-slave system for minimally invasive surgery," Proc. IEEE Intl. Conf. Rob. \& Auto., pp. 371-376, 2004.

[5] M. C. Cavusoglu, D. Feygin, and F. Tendick, "A critical study of the mechanical and electrical properties of the phantom haptic interface and improvements for high 
performance control," PRESENCE Rev., vol. 11, pp. 555-568, 2002.

[6] H. Mayeda, K. Osuka, and A. Kangawa, "A new identification method for serial manipulator arms," Proc. 9th IFAC World Congress, pp. 2429-2434, 1984.

[7] H. Mayeda, K. Yoshida, and K. Osuka, "Base parameter of manipulator dynamic models," Proc. IEEE Int. Conf. Rob. \& Auto., pp. 312-321, 1988.

[8] Y. Nakamura and M. Ghodoussi, "Base parameter analysis of open and closed link mechanism using covariance matrix of nonlinearity," Proc. IEEE Int. Conf. Rob. \& Auto., pp. 294-302, 1989.

[9] P. K. Khosla and T. Kanade, "Parameter identification of robot dynamics," Proc. IEEE Conf. Dec. \& Cont., pp. 1754-1760, 1985.

[10] K. Yoshida, N. Ikeda, and H. Mayeda, "Experimental identification methods for an industrial robot manipulator," IEEE/RSJ Intl. Conf. Intel. Rob. Systs., pp. 546$560,1991$.

[11] S. Tafazoli, P. D. Lawrence, and S. E. Salcudean, "Identification of inertial and friction parameters for excavator arms," Trans. IEEE Rob. \& Auto., vol. 15, pp. 966-971, 1999.

[12] K. J. Astrom and B. Wittenmark, Adaptive Control. Addison-Wesley Longman Publishing Co., Inc., 1994.

[13] P. Hsu, P. M. Bodson, S. Sastry, and B. Paden, "Adaptive identification and control of manipulators without using joint accelerations," Proc. IEEE Intl. Conf. Rob. \& Auto., pp. 1210-1215, 1987.

[14] K. Otani, T. Kakizaki, and K. Kogure, "Dynamic parameter identification of an industrial robot and its application to trajectory controls," IEEE/RSJ Intl. Conf. Intel. Rob. Systs., pp. 990-997, 1992.

[15] P. Abolmaesumi, K. Hashtrudi-Zaad, D. Thompson, and A. M. Tahmasebi, "A haptic-based system for medical examination," Proc. IEEE Intl. Conf. Engineering in Medicine \& Biology, pp. 1853-1856, 2004. 